\title{
CARGA DE ENFERMEDAD ATRIBUIBLE AL USO DEL TABACO EN PARAGUAY Y POTENCIAL IMPACTO SANITARIO Y ECONÓMICO DEL AUMENTO DEL PRECIO A TRAVÉS DE IMPUESTOS
}

\author{
Ariel Bardach ${ }^{1, a}$, Felicia Cañete ${ }^{2, b}$, Víctor Guillermo Sequera ${ }^{2, b}$, Alfredo Palacios $^{1, \mathrm{c}}$, Andrea Alcaraz $^{1, \mathrm{~d}}$, \\ Belén Rodríguez ${ }^{1, \mathrm{e}}$, Joaquín Caporale ${ }^{1, \mathrm{f}}$, Federico Augustovski ${ }^{1, a}$, Andrés Pichon-Riviere $^{1, a}$
}

\begin{abstract}
RESUMEN
Objetivos. Estimar la carga de enfermedad asociada al consumo de tabaco en Paraguay y evaluar el potencial efecto económico y sanitario del aumento de precio mediante impuestos. Materiales y métodos. Se diseñó un modelo de microsimulación de Monte Carlo que incorporó la historia natural, costos y calidad de vida de enfermedades asociadas al tabaquismo para el 2015. Asimismo, se estimó el impacto en varios escenarios de aumento de impuestos sobre la prevalencia de tabaquismo y la recaudación fiscal. Resultados. 3354 personas mueren al año en Paraguay por consecuencia del tabaquismo. El $19 \%$ de las muertes son por enfermedad isquémica cardíaca, el $15 \%$ por accidentes cerebrovasculares. El $77 \%$ de las muertes por enfermedad pulmonar obstructiva crónica y el $83 \%$ de cáncer de pulmón son atribuibles al tabaquismo. Estas enfermedades en Paraguay representan un costo médico directo anual de más de $1,5 \times 10^{6}$ millones de guaraníes, mientras la recaudación impositiva por la venta de cigarrillos apenas llega a cubrir un $20 \%$ de este gasto. Un aumento en el precio de los cigarrillos del $50 \%$ vía impuestos, podría llevar a evitar 2507 muertes en diez años y generar recursos por 2,4 × $10^{6}$ millones por ahorro en gastos sanitarios y aumento de recaudación. Conclusiones. El costo y la carga de enfermedad asociado al consumo de tabaco en el sistema de salud es elevado en Paraguay. Un aumento del precio de los cigarrillos a través de los impuestos tendría importantes beneficios sanitarios y podría compensar parcialmente los costos sanitarios.
\end{abstract}

Palabras clave: Tabaquismo; Costo de enfermedad; Evaluación de costo-efectividad; Impuestos; Paraguay (fuente: DeCS BIREME).

\section{BURDEN OF DISEASE ATTRIBUTABLE TO TOBACCO USE IN PARAGUAY, AND POTENTIAL HEALTH AND FINANCIAL IMPACT OF INCREASING PRICES THROUGH TAXING}

\begin{abstract}
Objectives. To consider the burden of disease associated to tobacco consumption in Paraguay and to evaluate the potential economic and health effect of price increase through taxes. Materials and Methods. A Monte Carlo simulation model was designed incorporating natural history, costs, and quality of life of diseases associated to smoking for 2015. Also, several scenarios were considered for the impact of tax raises on the prevalence of smoking and fiscal collection. Results. In Paraguay, 3,354 people die every year as a consequence of smoking. Nineteen percent of deaths are due to cardiac ischemia, $15 \%$ due to stroke. $77 \%$ of deaths due to chronic obstructive pulmonary disease (COPD), and $83 \%$ of lung cancer can be attributed to smoking. These diseases in Paraguay represent an annual direct medical cost of more than 1.5 trillion PYG, while the tax collection from cigarette sales barely covers $20 \%$ of this expense. A $50 \%$ increase in the price of cigarettes via taxes could avoid 2507 deaths in ten years and generate resources by 2.4 trillion in savings in health expenses and tax of collection. Conclusions. The cost and the burden of disease associated to tobacco consumption is high in the health system in Paraguay. An increase in cigarette price through taxes could have significant health benefits and could offset health costs in part.
\end{abstract}

Keywords: Tobacco use; Cost of illness; Cost-Effectiveness evaluation; Taxes; Paraguay (source: MeSH NLM)

\footnotetext{
Instituto de Efectividad Clínica y Sanitaria (CONICET). Buenos Aires, Argentina

Dirección de Vigilancia de Enfermedades No Transmisibles, Ministerio de Salud Pública y Bienestar Social. Asunción, Paraguay

Médico, doctor en Salud Pública; ${ }^{\text {b }}$ médico, especialista en Medicina Interna, magíster en Salud Pública; ' licenciado en Economía, magíster en Economía; médica especialista en Cardiología, magíster en Efectividad Clínica; ${ }^{\mathrm{C}}$ médico especialista en Medicina Interna, magíster en Efectividad Clínica; ${ }^{\mathrm{f}}$ licenciado en Economía, magíster en Finanzas Públicas Provinciales y Municipales

Recibido: 02/06/2018 Aprobado: 28/11/2018 En línea: 21/12/2018
}

Citar como: Bardach A, Cañete F, Sequera VG, Palacios A, Alcaraz A, Rodríguez B, et al. Carga de enfermedad atribuible al uso del tabaco en Paraguay y potencial impacto sanitario y económico del aumento del precio a través de impuestos. Rev Peru Med Exp Salud Publica. 2018;35(4):599-609. doi: 10.17843/rpmesp.2018.354.3708 


\section{INTRODUCCIÓN}

El consumo de productos del tabaco es la principal causa de enfermedad y muerte que podría prevenirse a nivel global. El 6,3\% de las muertes prematuras ocurridas en el mundo, así como de años de vida ajustados por discapacidad (AVAD) entre 1990 y 2010 podrían ser consecuencia del consumo de tabaco. Seis millones de muertes por año se deben a las enfermedades derivadas del consumo de tabaco como cáncer, enfermedad cardiovascular y enfermedad pulmonar obstructiva crónica (EPOC) ${ }^{(1,2)}$

En Latinoamérica, el tabaquismo ocupa el quinto lugar como factor de riesgo de acuerdo a muertes y AVAD perdidos ${ }^{(1)}$. Su uso se vincula a menor productividad y a un impacto importante en el gasto de hogares, y el incremento de la pobreza de los individuos ${ }^{(3)}$. Esta adicción origina además un impacto considerable en los costos del sistema de salud. En Latinoamérica, cada año de consumo de tabaco es responsable de cerca de 34000 millones de dólares en costos directos para el sistema de salud. Esto corresponde al $0,7 \%$ del producto interno bruto (PIB) de Latinoamérica y al $8,3 \%$ del total del presupuesto en salud ${ }^{(4)}$.

En Paraguay, la prevalencia del tabaquismo ronda entre el $19,9 \%-25,9 \%$ en hombres y $5,0 \%-7,4 \%$ en mujeres, representando una de las más bajas de Sudamérica ${ }^{(5)}$. Según la Encuesta Global de Tabaquismo en jóvenes Paraguay 2014, el $7 \%$ de los estudiantes entre 13 y 15 años refirió haber consumido alguna forma de tabaco en los últimos 30 días, siendo este problema mayor en los niños $(7,4 \%)$ que en las niñas $(6,6 \%)^{(6)}$.

Con el objetivo de controlar el tabaquismo a nivel global, la Organización Mundial de la Salud (OMS) generó el Convenio Marco para el Control del Tabaco (CMCT) suscrito en junio de 2003 y ratificado en septiembre de 2006 por Paraguay ${ }^{(7)}$. Dentro del cumplimiento de dicho marco, el gobierno paraguayo promulgó el 2015 la Ley 5538, que estableció la impresión de advertencias sanitarias visuales en el $40 \%$ de la cara principal de la cajetilla, reguló la publicidad, la venta y el consumo de tabaco en lugares públicos y de trabajo, y aumentó el impuesto al consumo del tabaco. Si bien la ley representa un progreso para el avance en el control del tabaquismo, Paraguay continua siendo el país con la oferta más barata y accesible a cigarrillos en toda América ${ }^{8}$. El precio de la marca más popular y de la marca premium en 2014 fue de 0,54 y 3,06 dólares internacionales, respectivamente ${ }^{(9)}$,

La demanda de productos del tabaco está muy influenciada por el precio y por el ingreso económico, ya que se incrementa a medida que aumenta el poder adquisitivo. Este fenómeno se observa en países en desarrollo, en los que el nivel de ingreso de la población ha aumentado más rápidamente que el precio de los cigarrillos, haciéndose

\section{MENSAJES CLAVE}

Motivación para realizar el estudio. Obtener información sobre la carga de enfermedad vinculada al tabaquismo en Paraguay y evaluar el potencial efecto económico y sanitario del aumento de impuestos.

Principales hallazgos. Casi 3500 personas mueren cada año en Paraguay como consecuencia del tabaquismo. Estas enfermedades representan un costo médico directo anual de más de 1,5 billones de guaraníes, mientras la recaudación impositiva por la venta de cigarrillos apenas llega a cubrir un $20 \%$ de este gasto.

Implicancias. Un aumento en el precio del $50 \%$ vía impuestos podría evitar 2507 muertes en diez años y generar recursos por $2,4 \times 10^{6}$ millones de guaraníes por ahorro.

estos más asequibles con el tiempo. El aumento de precios de los cigarrillos a través de los impuestos es una medida costo-efectiva y eficaz para controlar el tabaquismo, ya que desalienta el consumo de tabaco afectando principalmente el inicio en jóvenes, la probabilidad de re-incidencia de ex-fumadores y reduce el consumo entre los fumadores, disminuyendo de esta manera la carga de enfermedad y el coste sanitario ${ }^{(10-13)}$. La política tributaria debe acompañarse de medidas adicionales para la reducción de la asequibilidad, como por ejemplo ajustes inflacionarios en forma automática.

La información presentada en este artículo surge de una evaluación económica basada en un modelo matemático en salud. Este tipo de estudios es cada vez más utilizado por decisores de salud para informar políticas relevantes a la hora de establecer los recursos. En tal sentido, este estudio pretende estimar la carga de enfermedad relacionada al consumo de tabaco y evaluar el impacto en salud y económico, que podrían tener los diferentes escenarios de aumento de los impuestos a cigarrillos en Paraguay.

\section{MATERIALES Y MÉTODOS}

El estudio consiste en una evaluación económica realizada a través de un modelo de simulación programado de novo a partir de fuentes secundarias de datos nacionales. El mismo es parte de un proyecto colaborativo de centros de investigación e instituciones sanitarias de varios países latinoamericanos para estimar el impacto del tabaquismo en Paraguay sobre varios resultados en salud pública y financieros. Cuenta con un módulo de carga de enfermedad y otro módulo económico de evaluación del impacto en salud y la recaudación impositiva ante tres escenarios posibles de incremento de precios de cigarrillos. Este modelo fue desarrollado por investigadores y decisores en salud procedentes de universidades, institutos de investigación y ministerios de más de siete países de Latinoamérica. 
Una presentación más detallada del modelo se puede encontrar en el artículo de Pichon-Riviere et al. (14), en publicaciones que utilizaron o evaluaron el modelo ${ }^{(15-18)}$, y en reportes que presentan datos sobre la carga de enfermedad para cada uno de los países, disponibles en el sitio web www.iecs.org.ar/tabaco.

\section{CARGA DE ENFERMEDAD}

Se utilizó la técnica de microsimulación de Monte-Carlo de primer orden, programada en Microsoft Excel@ 2003 (Microsoft, Redmond, WA). El modelo incluye la historia natural, los costos y la calidad de vida relacionada a la salud de enfermedades asociadas al tabaquismo en adultos: enfermedad coronaria y no coronaria del corazón, neumonía, enfermedad pulmonar obstructiva crónica (EPOC), accidente cerebrovascular (ACV), cáncer de pulmón y otras neoplasias, diez en total. Se estimó el riesgo basal de dichas condiciones en personas no fumadoras. Luego de la simulación a nivel individual de cohortes hipotéticas de sujetos, se estimó el riesgo de que ocurra cada tipo de evento, de progresión de enfermedad y de muerte, según características demográficas, clínicas y ecuaciones específicas del riesgo. Los resultados obtenidos fueron número de muertes o eventos y los costos relacionados al tabaquismo, disminución de la esperanza de vida y de los años de vida ajustados por la calidad (AVAC), así como la cantidad de AVAD.

Para datos de incidencia se realizó la aproximación metodológica aplicada en varios modelos epidemiológicos y económicos de la OMS ${ }^{(19-21)}$. La incidencia poblacional según edad y género de los eventos agudos fue calculada con la fórmula:

$$
R_{\text {pob }}=\frac{R_{\text {muerte }}}{L}
$$

donde $R_{\text {muerte }}$ equivale a la tasa de mortalidad, $L$ a letalidad calculada del evento. La incidencia anual en no fumadores es:

$$
R_{\text {no-fum }}=\frac{R_{\text {pob }}}{\left(R R_{\text {fum }}{ }^{*} f_{\text {fum }}\right)+\left(R R_{\text {ex-fum }}{ }^{*} f_{\text {ex-fum }}\right)+f_{\text {no-fum }}}
$$

donde $R_{\text {pob }}$ es incidencia poblacional, $R R_{\text {fum }}$ y $R R_{\text {ex-fum }}$ equivalen a los riesgos relativos del evento en fumadores y exfumadores, y f representa las fracciones de cada categoría de tabaquismo. En el caso de las enfermedades neoplásicas, para incorporar información de sobrevida, la incidencia por grupos de edad (i) fue estimada a partir de la siguiente ecuación:

$$
\operatorname{Rdx}_{i}=\left(\sum_{n=0}^{10} R m_{i} \times P_{n}\right) \times \frac{1}{1-S_{10}}
$$

en donde $\mathrm{Rm}_{\mathrm{i}}$ equivale a la tasa de mortalidad en la edad $i+n ; P_{n}$ es la probabilidad condicional de morir al año $n$ posterior al diagnóstico de la enfermedad, y $\mathrm{S}_{10}$ es la proporción de supervivientes posterior al año 10. Cuando no existió información suficiente para utilizar esta ecuación, la incidencia fue estimada directamente a partir de datos arrojados por el proyecto Globocan ${ }^{(20)}$.

\section{CALIBRACIÓN Y VALIDACIÓN}

La calibración del modelo se realizó mediante comparación de tasas específicas de mortalidad que fueron predichas por el modelo para cada grupo de edad y de género, con las procedentes de las estadísticas vitales. Las variaciones se consideraron aceptables cuando eran inferiores al $10 \%$ $y$, en caso de que fueran mayores, se transformaron las ecuaciones de riesgo para obtener estimaciones dentro de los rangos deseados. Se realizó la validación externa confrontando resultados de incidencia y mortalidad predichos por el modelo con aquellos reportados en distintos estudios epidemiológicos y clínicos ajenos al modelo, para las mismas condiciones clínicas.

\section{FUENTES DE DATOS}

Los datos epidemiológicos del modelo de incidencia, así como los demográficos proceden de informes oficiales de Paraguay (Tabla 1) ${ }^{(22)}$. La estimación de la letalidad en las enfermedades cardiovasculares se consideró igual que la observada para Argentina, validada por el equipo local (23). Para la letalidad en cáncer, se utilizó Globocan ${ }^{(20)}$. La prevalencia de tabaquismo se obtuvo de la Encuesta Nacional de Factores de Riesgo de Paraguay, del $2011^{(5)}$.

\section{COSTOSDELOS SERVICIOSDESALUDYPERSPECTIVA DEL ESTUDIO}

La perspectiva que utiliza nuestro estudio es la del sistema de salud. Es por ello que se incluyeron los costos médicos del sistema de salud, y no se consideraron otros costos de otras perspectivas (costos del paciente y la familia, pérdida de productividad, etc). El modelo de costo-efectividad incluye 29 indicadores de costos con relación al atendimiento clínico directo de distintas enfermedades asociadas al tabaquismo. Para ello, se estableció una metodología común que contempló calcular costos de los eventos o enfermedades a través una perspectiva de macro-costeo o de micro-costeo, conforme a la calidad y la disponibilidad de los datos.

Debido a dificultades en el acceso a la información del sector público, se decidió avanzar en una estimación de costos de eventos utilizando información proveniente del sector privado de salud. De acuerdo con ello, el equipo local mantuvo reuniones de trabajo con los referentes de uno de los hospitales privados de mayor escala de atención de la ciudad de Asunción. Se obtuvieron estimaciones de costos para los siguientes eventos de salud: ACV, infarto agudo de miocardio (IAM), y su seguimiento anual, neumonía, 
Tabla 1. Datos demográficos, epidemiológicos, de costos e impositivos incluidos en el modelo de simulación económica para estimar carga de enfermedad asociada al consumo de tabaco en Paraguay, 2015

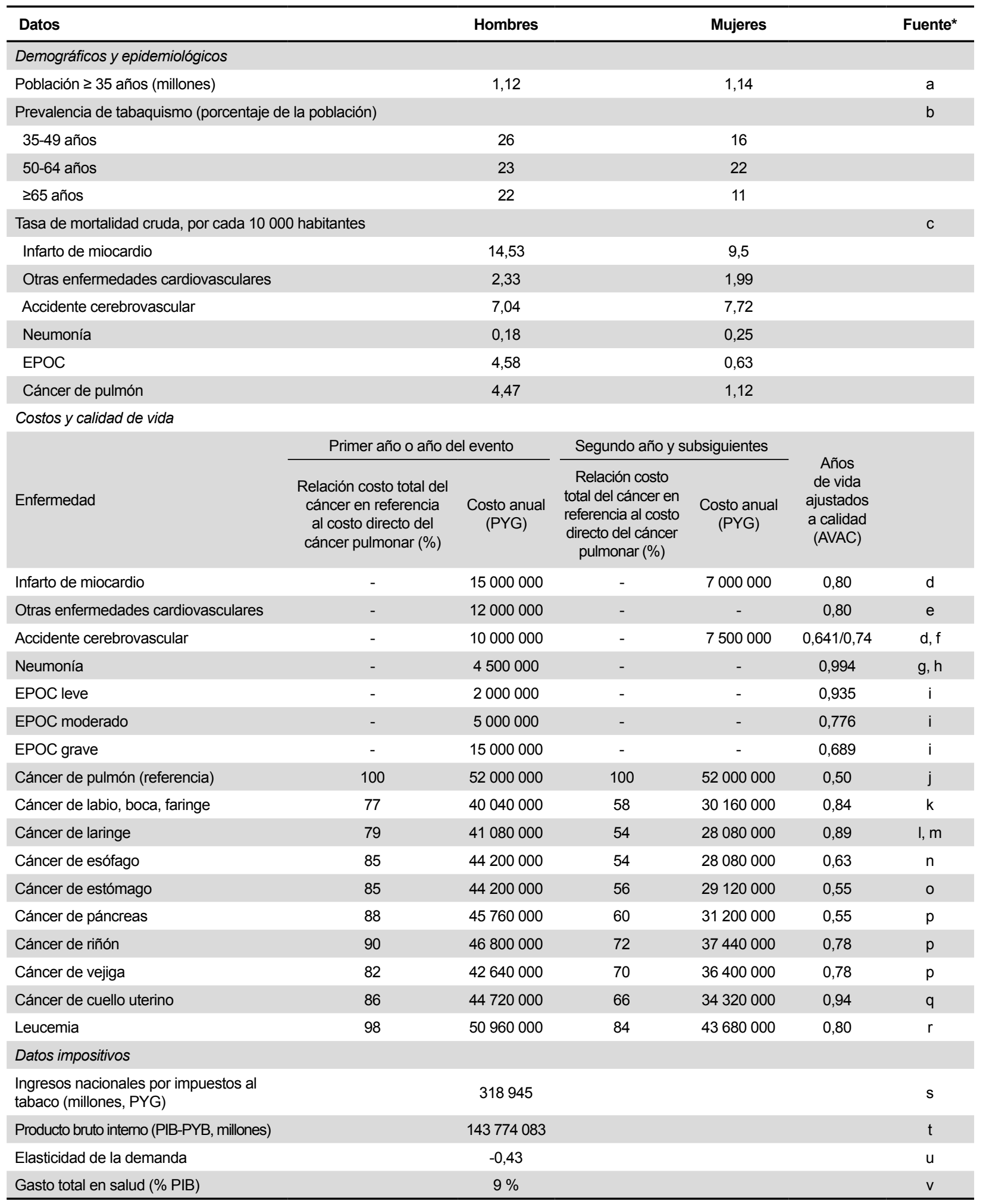

Tasa de cambio: 1 USD $=5$ 787guaraníes (diciembre 2015)

PYG: guaraníes, USD: dólares estadounidenses, EPOC: enfermedad pulmonar obstructiva crónica

${ }^{*}$ Anexo 2 
EPOC, cáncer de pulmón de primer año, segundo año y consecutivos.

Los costos de los eventos de cáncer fueron valuados de acuerdo a la estimación hecha para el primer y segundo año de cáncer de pulmón. Para esto se realizó un consenso, cuyo grupo de trabajo estuvo conformado por cinco expertos locales en el área de oncología provenientes de diferentes instituciones del sector público y privado. A este grupo se le solicitó calcular la relación entre cada uno de los costos médicos totales para la atención de cada tipo cáncer y el costo médico total del cáncer de pulmón. Se les aclaró a los expertos que en sus respuestas consideraran todos los costos relacionados con la enfermedad en el primer o segundo año (diagnóstico, cirugías, quimioterapia, internaciones, consultas, radioterapia, cuidados paliativos, etc.) y para un paciente «promedio» con cada patología.

\section{IMPACTO DE POLÍTICAS FISCALES}

El impacto del aumento de impuestos al cigarrillo fue revisado en niveles: 1) Sobre la prevalencia de tabaquismo y eventos sanitarios; 2) Sobre la recaudación de impuestos; y 3) Impacto financiero general. Se tomaron en cuenta como medidas puntuales: la elasticidad del precio de la demanda, cambios en los niveles de consumo y los cambios en los precios.

El impacto que era esperado para los diferentes contextos de aumento de impuestos se calculó de la siguiente manera: Prevalencia ${ }_{\text {pre }}=$ prevalencia de fumadores antes del aumento de precio; $\mathrm{E}_{\mathrm{d}}=$ elasticidad precio de la demanda; $\Delta \% \mathrm{P}=$ diferencia en porcentaje del precio; $\mathrm{e} \mathrm{I}_{\mathrm{p}}=$ proporción del cambio en el consumo, con consecuencias en la prevalencia del ser fumador actual.

Prevalencia $_{\text {post }}=$ Prevalencia $_{\text {pre }}+\left(\mathrm{E}_{\mathrm{d}} * \Delta \% \mathrm{P} * \mathrm{I}_{\mathrm{p}} *\right.$ Prevalencia $\left._{\text {pre }}\right)$

Para la traslación del aumento de impuestos al impacto en el consumo y el riesgo, se calcularon en tres contextos diferentes:

El primero a corto plazo: donde se asume que la mitad de la disminución del consumo impacta en la prevalencia $\left(I_{p}=0,5\right)$ y esta disminución se transfiere a la población de exfumadores.

A mediano plazo: incluye a los efectos hipotéticos relacionados con disminuir el número de cigarrillos consumidos. Esta disminución fue considerada en forma proporcional a la disminución del consumo, al $75 \%$ del exceso del riesgo de diferencia entre un tabaquista actual y un extabaquista, asumiendo que $25 \%$ del riesgo adicional, sería eliminado cuando la persona pasa a ser un exfumador ${ }^{(24)}$.

A largo plazo: el máximo efecto, el cual podría aparecer en diez años. Es igual al escenario previo, aunque con efecto mayor en la prevalencia $\left(I_{p}=0,75\right)$, y en la cual los exfumadores se mantienen constante respecto al contexto de base y toda la disminución en la prevalencia se pasa a los no tabaquistas.

Para valuar el caso basal, se combinaron resultados de los tres contextos (desde que se inicia la política hasta los siguientes diez años) de la siguiente manera: del año 1 al año cinco se asigna una evolución lineal del contexto de corto a mediano plazo; del año seis al diez la evolución es lineal desde mediano a largo plazo.

\section{IMPACTO EN LA RECAUDACIÓN IMPOSITIVA}

La variación en la recaudación impositiva a esperarse en los diferentes contextos de incremento de precios de cigarrillos, fue estimada de la manera siguiente:

$$
\Delta \% \mathrm{R}=(1+\Delta \% \text { consumo }) *\left(1+\frac{\Delta \% \text { precio }}{\% \text { impuesto }}\right)-1
$$

Donde $\Delta \%$ R es el cambio en el porcentaje de la recaudación; $\Delta \%$ consumo es el cambio en porcentaje del consumo a esperarse por el incremento del precio de venta; $\Delta \%$ precio es el cambio en el porcentaje del precio de la venta; y \%impuesto es la proporción del precio inicial de venta al público que se relaciona a la carga impositiva.

\section{IMPACTO ECONÓMICO GENERAL}

Estimado a través de la combinación de la variación del gasto sanitario directo que es atribuible al consumo de tabaco calculado en el primer punto con la variación en la recaudación por los impuestos.

\section{RESULTADOS}

\section{CALIBRACIÓN Y VALIDACIÓN}

Una vez finalizado el proceso de calibración o validación interna, la tasa media de cada evento prevista por el modelo se encontró dentro del $+/-4 \%$ de aquellas reportadas oficialmente para el país, lo cual muestra el alto grado de correlación. En la Figura 1 pueden observarse las predicciones del modelo, en comparación con las estadísticas nacionales y Globocan. La validación externa mostró una muy buena correlación entre los resultados previstos por el modelo y en los estudios epidemiológicos observados.

\section{MUERTES Y EVENTOS ATRIBUIBLES AL TABAQUISMO}

En 2015, el cigarrillo fue el responsable de 3354 fallecimientos en Paraguay. Esto representa el $12 \%$ del total de los fallecimientos que ocurren al año. Del total de muertes por enfermedades cardíacas y de las producidas por ACV pueden atribuirse al tabaquismo el 
A)
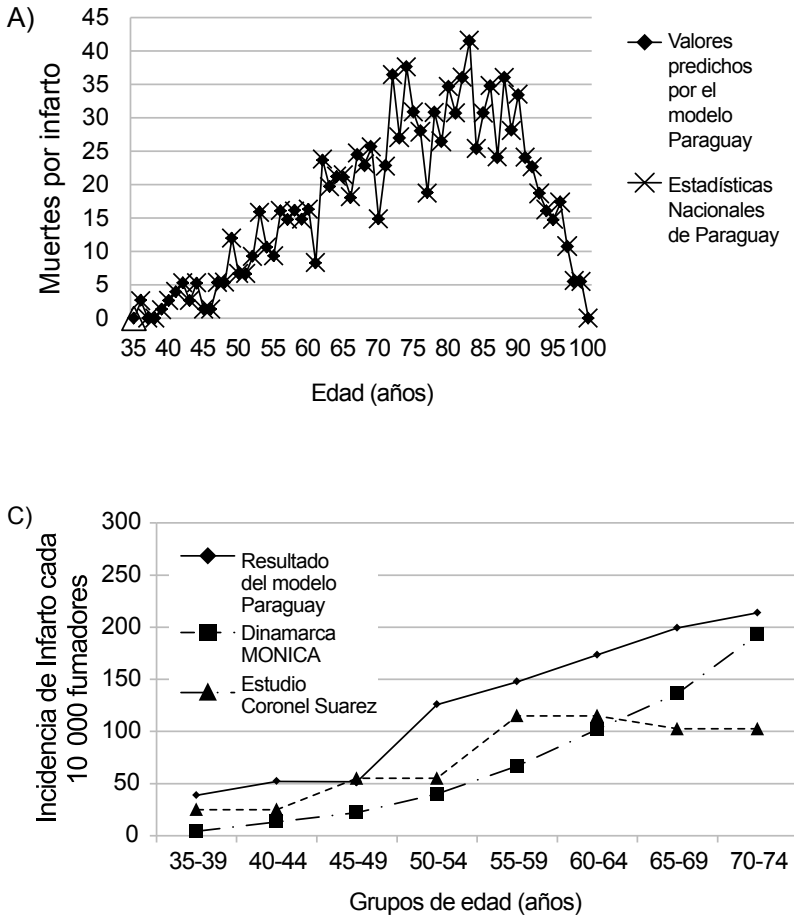

E)

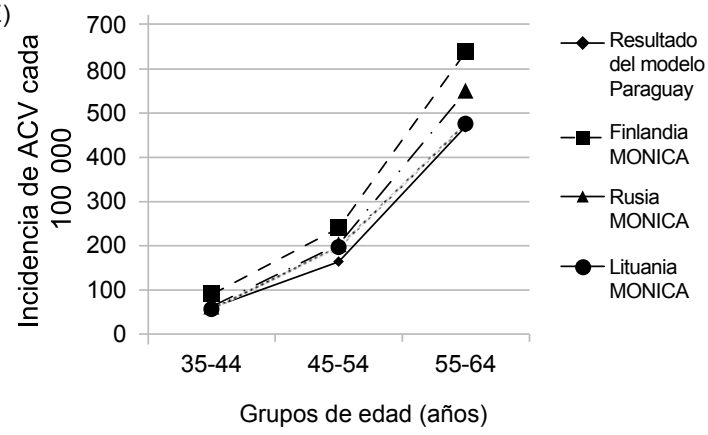

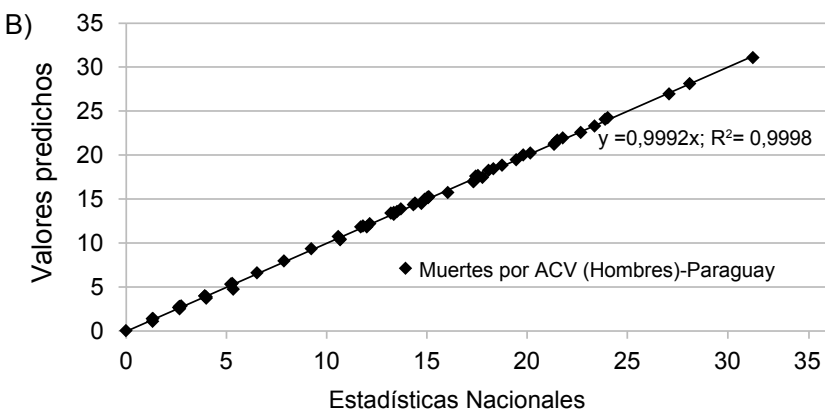

D)
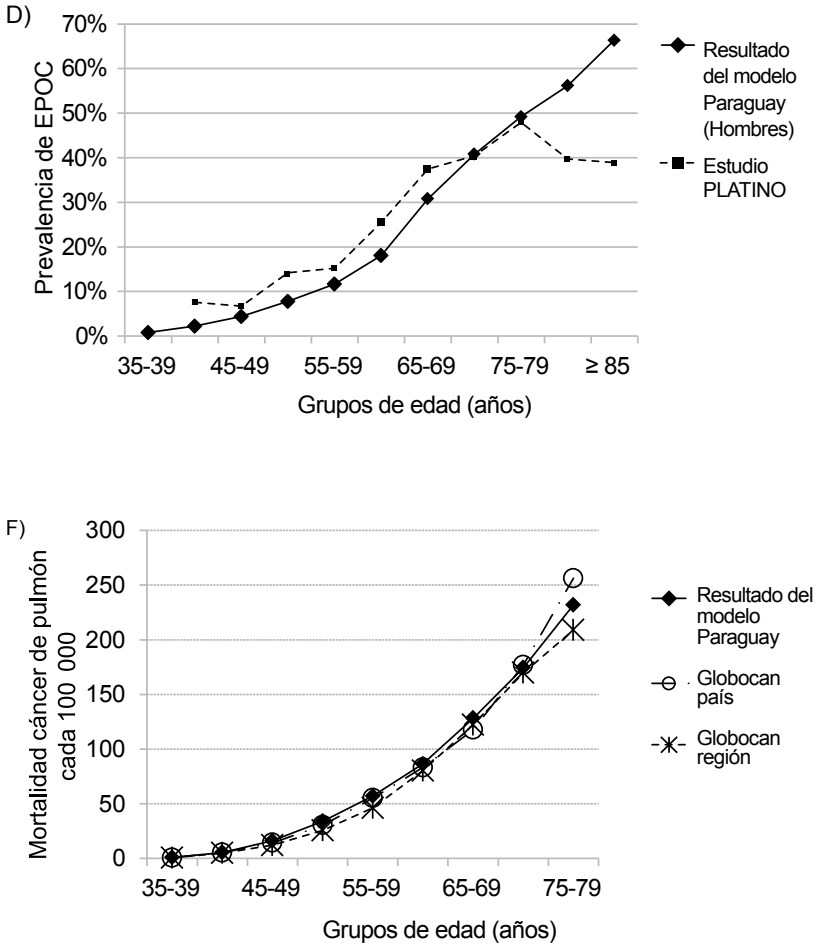

A. Calibración: Número anual de muertes predicho por el modelo comparado con las estadísticas nacionales y Globocan. Ejemplo: Infarto de miocardio en mujeres

B. Correlación entre los valores predichos por el modelo y los valores esperados de acuerdo a estadísticas nacionales. Ejemplo: muertes por accidente cerebro vascular en hombres

Validación externa con estudios epidemiológicos seleccionados en hombres (C, D, E, F)

C. Incidencia de infarto predicho por el modelo comparado con el estudio de incidencia de base poblacional: Danish WHO MONICA study register, (EM27) y Estudio de incidencia de infarto en Argentina (Coronel Suarez) (EM29,30)

D. Prevalencia de EPOC predicha por el modelo comparado con la prevalencia reportada por el estudio Platino (PLATINO Latin American Project for the Investigation of Obstructive Lung Disease) (EM31)

E. Incidencia de Accidente Cerebro Vascular (ACV) predicha por el modelo comparado con el WHO MONICA study register en países seleccionados (Finland WHO MONICA study register North Karelia province, Russia WHO MONICA study register Novosibirsk city, Lithuania WHO MONICA study register Kaunas city) (EM28)

F. Tasa de mortalidad por cáncer de pulmón predicha por el modelo comparado con las estimaciones realizadas por la International Agency for Research on Cancer (IARC) (EM16,17)

Las referencias bibliográficas mencionadas corresponden al estudio metodológico (EM) publicado por Pichon-Riviere et al. ${ }^{(14)}$

Figura 1. Consistencia interna, calibración y validación externa del modelo de simulación de carga de enfermedad en Paraguay, 2015

$19 \%$ y el $15 \%$, respectivamente. Estos valores son más elevados en las patologías respiratorias como EPOC $(77 \%)$ y cáncer pulmonar (83 \%). También, el $16 \%$ de las neumonías y el $29 \%$ de las muertes por otros tipos de cánceres se atribuyen al hábito tabáquico (Tabla 2).
Anualmente, el consumo de tabaco es responsable de 22386 eventos en el país. De estos, 9075 corresponden a infartos y otras enfermedades cardíacas, 1414 a ACV, 1410 a nuevos diagnósticos de cáncer por año y 10385 a EPOC. La fracción atribuible al cáncer es de $83 \%$ para 
Tabla 2. Muertes, eventos y costos asociados y atribuibles al tabaquismo en Paraguay, 2015

\begin{tabular}{|c|c|c|c|c|c|c|c|c|c|}
\hline \multirow[t]{2}{*}{$\begin{array}{l}\text { Patología asociada al } \\
\text { tabaquismo }\end{array}$} & \multirow[t]{2}{*}{$\begin{array}{l}\text { Muertes } \\
\text { totales }\end{array}$} & \multicolumn{2}{|c|}{$\begin{array}{l}\text { Muertes } \\
\text { Atribuibles }\end{array}$} & \multirow[t]{2}{*}{$\begin{array}{l}\text { Eventos } \\
\text { totales }\end{array}$} & \multicolumn{2}{|c|}{$\begin{array}{l}\text { Eventos } \\
\text { atribuibles }\end{array}$} & \multirow[t]{2}{*}{$\begin{array}{c}\text { Costos } \\
\text { totales } \\
\text { (millones de } \\
\text { PYG) }\end{array}$} & \multicolumn{2}{|c|}{$\begin{array}{c}\text { Costos } \\
\text { atribuibles } \\
\text { (millones de } \\
\text { PYG) }\end{array}$} \\
\hline & & $\mathbf{n}$ & $\%$ & & $\mathbf{n}$ & $\%$ & & $\mathbf{n}$ & $\%$ \\
\hline Infarto agudo de miocardio (IAM) & 2870 & 573 & 20 & 18959 & 5077 & 27 & \multirow{3}{*}{$2736683^{\dagger}$} & \multirow{3}{*}{$641603^{\dagger}$} & \multirow{3}{*}{23} \\
\hline Síndrome coronário agudo no IAM & 519 & 94 & 18 & 17669 & 3972 & 22 & & & \\
\hline $\begin{array}{l}\text { Muerte cardiovascular de causa } \\
\text { no isquémica }\end{array}$ & 184 & 26 & 14 & 184 & 26 & 14 & & & \\
\hline Accidente cerebrovascular & 1770 & 270 & 15 & 8433 & 1414 & 17 & 573502 & 108257 & 19 \\
\hline Cáncer de pulmón & 728 & 607 & 83 & 772 & 644 & 83 & 108246 & 90526 & 74 \\
\hline Neumonía & 52 & 8 & 16 & 625 & 102 & 16 & 2814 & 458 & 16 \\
\hline $\begin{array}{l}\text { Enfermedad pulmonar obstructiva } \\
\text { crónica }\end{array}$ & 1174 & 907 & 77 & 14887 & 10385 & 70 & 595775 & 440400 & 84 \\
\hline Cáncer de boca y faringe & 39 & 26 & 67 & 105 & 71 & 68 & & & \\
\hline Cáncer de esófago & 158 & 108 & 68 & 170 & 115 & 68 & & & \\
\hline Cáncer de estómago & 314 & 75 & 24 & 409 & 97 & 24 & & & \\
\hline Cáncer de páncreas & 285 & 59 & 21 & 296 & 61 & 21 & & & \\
\hline Cáncer de riñón & 52 & 16 & 31 & 90 & 30 & 33 & $381732^{\ddagger}$ & $107000^{\ddagger}$ & 28 \\
\hline Cáncer de laringe & 139 & 115 & 82 & 280 & 232 & 83 & & & \\
\hline Leucemia & 211 & 39 & 18 & 228 & 42 & 18 & & & \\
\hline Cáncer de vejiga & 44 & 20 & 45 & 143 & 64 & 45 & & & \\
\hline Cáncer de cuello de útero & 426 & 27 & 6 & 865 & 55 & 6 & & & \\
\hline Tabaquismo pasivo y otras causas & 386 & 386 & 100 & NC & NC & NC & NC & 180472 & 100 \\
\hline $\begin{array}{l}\text { Total por patologías asociadas al } \\
\text { tabaquismo }\end{array}$ & 9351 & 3354 & 36 & 64115 & 22386 & 35 & 4398752 & 1568716 & 36 \\
\hline
\end{tabular}

pulmón y laringe, de $68 \%$ en los de boca o faringe y en cáncer de esófago, siendo además importante en cáncer de estómago, renal y de páncreas (Tabla 2).

El impacto difiere para ambos sexos: en hombres, 37,4\% de las muertes y $45 \%$ de los eventos por enfermedades son atribuibles al tabaquismo; y en mujeres, dichos valores son $30,4 \%$ y $20 \%$, respectivamente.

\section{AÑOS DE VIDA PERDIDOS Y CALIDAD DE VIDA ASOCIADOS AL CONSUMO DE TABACO}

Los varones tabaquistas tienen una esperanza de vida 5,56 años menor que los que no tienen el hábito, mientras que en los extabaquistas la reducción es de 2,4 años. En las mujeres ocurre también algo similar, siendo la esperanza de vida entre fumadoras 6,05 años menor que en no tabaquistas y 2,22 en las exfumadoras con respecto a las que no son fumadoras.
Los años de vidas perdidos (AVP) por consumo de tabaco por año fueron 78 295, el $67 \%$ de los AVP fue por muertes prematuras y $33 \%$ por vivir en condiciones subóptimas en cuanto a la calidad de vida relacionada en salud. Del total de estos AVP, 18238 se pierden en mujeres y más del triple (60 056) se pierden en hombres.

Si se toma en cuenta al daño por humo ajeno y otras condiciones no incluidas, como la enfermedad perinatal o accidentes vinculados al hecho de fumar, la cifra alcanza a 88473 AVP por año.

\section{COSTOS ASOCIADOS CON EL TABAQUISMO}

En Paraguay, nuestros resultados sugieren que el consumo de tabaco es responsable anualmente de costos médicos directos de aproximadamente $1,5 \times 10^{6}$ millones de guaraníes (PYG), lo que corresponde al 1,09\% del producto interno bruto (PIB) nacional y al $12,12 \%$ del gasto anual en salud pública. La recaudación en impuestos por 
Tabla 3. Impacto en la salud e impositivo acumulado a diez años con tres escenarios de aumento de precio de cigarrillos a través de impuestos en Paraguay, 2015

\begin{tabular}{lccc}
\hline \multirow{2}{*}{ Impacto en la salud e impositivo } & \multicolumn{3}{c}{ Aumento de precio } \\
\cline { 2 - 4 } Muertes evitadas & $10 \%^{\dagger}$ & $20 \%$ & $50 \%$ \\
\hline Enfermedades cardíacas evitadas & 501 & 1003 & 2507 \\
\hline Accidentes cerebrovasculares evitados & 1477 & 2953 & 7383 \\
\hline Nuevos casos de cáncer evitados & 422 & 845 & 1056 \\
Años de vida debidos a muerte prematura y discapacidad evitados & 13507 & 423 & 67537 \\
\hline Costos sanitarios evitados (millones PYG) & 229757 & 459514 & 114786 \\
\hline Aumento en la recaudación impositiva (millones PYG) & 349897 & 656027 & 1311810 \\
\hline Beneficio económico total (millones PYG) & 579655 & 1115542 & 2460596 \\
\hline
\end{tabular}

Tasa de cambio: 1 USD = 5787 guaraníes (diciembre 2015)

† El incremento del $10 \%$ en el precio de los cigarrillos se corresponde con un aumento del $16 \%$ del impuesto al tabaco

PYG: guaraníes

el consumo de cigarrillos en Paraguay ronda los 318945 millones anuales de PYG (estimaciones del 2015) ${ }^{(25)}$, un valor que llega a cubrir sólo al $20 \%$ de los gastos médicos directos causados por el cigarrillo al sistema nacional de salud.

Los factores principales de este elevado costo fueron las enfermedades cardiovasculares, así como las enfermedades neoplásicas, donde destaca el cáncer de pulmón y la enfermedad pulmonar crónica, debido a su alta fracción atribuible y a sus altos costos de tratamiento y seguimiento (Tabla 2). Los costos expresados en dólares se presentan en el Anexo 1.

\section{EFECTO POTENCIAL DEL INCREMENTO DE PRECIOS POR IMPUESTOS}

En la Tabla 3 y en la Figura 2 se presenta el impacto sanitario y financiero producido por aumentos de $10 \%, 20 \%$ y $50 \%$ en el precio de los paquetes de cigarrillos. A modo de ejemplo, el aumento en el precio de los cigarrillos en un $20 \%$ redunda en más de 1000 muertes y casi 3000 enfermedades cardíacas evitadas, con un costo sanitario evitado cercano a 460 mil millones de PYG. También se observa que ante el aumento del $50 \%$ del precio de cigarrillos se podría esperar a 10 años una reducción de muertes de $0,1 \%$. Los tres escenarios presentados de aumento de precio de cigarrillos fueron escogidos según plausibilidad y expectativas locales.

\section{DISCUSIÓN}

El presente estudio estima que en Paraguay el tabaquismo causa una importante carga al sistema de salud, expresada en morbilidad, mortalidad y costos. En el 2015 fue responsable del $12,2 \%$ de las muertes evitables en mayores de 35 años (3354 muertes por año) y generó una pérdida directa anual de más de 88473 años de vida saludable. Además, generó un costo directo de más de $1,5 \times 10^{6}$ millones, lo que equivale a 271 millones de dólares estadounidenses. Esta cifra representa el 1,1\% del producto interno bruto (PIB) y 12,1\% del gasto total en salud del país.

La recaudación impositiva estimada por el comercio de cigarrillos por el Ministerio de Hacienda para el 2016 fue alrededor de 318945 millones de guaraníes, un valor que apenas alcanza la quinta parte de los costos directos atribuibles al tabaquismo en el país ${ }^{(26)}$. El incremento del impuesto en un $50 \%$ al precio de los cigarrillos, una estrategia razonable y utilizada en otros países ${ }^{(27)}$, podría no solamente evitar una considerable cantidad de muertes y enfermedad, sino generar una recaudación que es equivalente a las pérdidas generadas por el tabaquismo anualmente en el país.

El único estudio sobre mortalidad atribuible realizado en Paraguay estimó, para el periodo 1998 - 2000, que el $13,4 \%$ de las muertes ocurridas entre los individuos de 35 y 65 años, fueron debidas al tabaquismo ${ }^{(26)}$. Este dato debería ser interpretado como muerte prematura atribuible por el rango de edad estudiado. El presente estudio amplía los datos a la morbilidad asociada, extiende la muerte atribuible a otros estratos superiores de la vida en donde el tabaco condiciona en mayor medida el estado de salud, y estima los costos directos asociados al tabaquismo. Esto último, fue consistente con otros trabajos a nivel global que estimaron la carga mundial, la posible reducción de su consumo y el impacto inherente a la subida de los impuestos ${ }^{(13,28,29)}$.

A pesar de que el tabaquismo sea la segunda droga lícita más consumida en Paraguay, las prevalencias de consumo de tabaco están entre las más bajas de la región. La Encuesta Nacional de Factores de Riesgos y Enfermedades No Trasmisibles del 2011 no muestra diferencias marcadas 

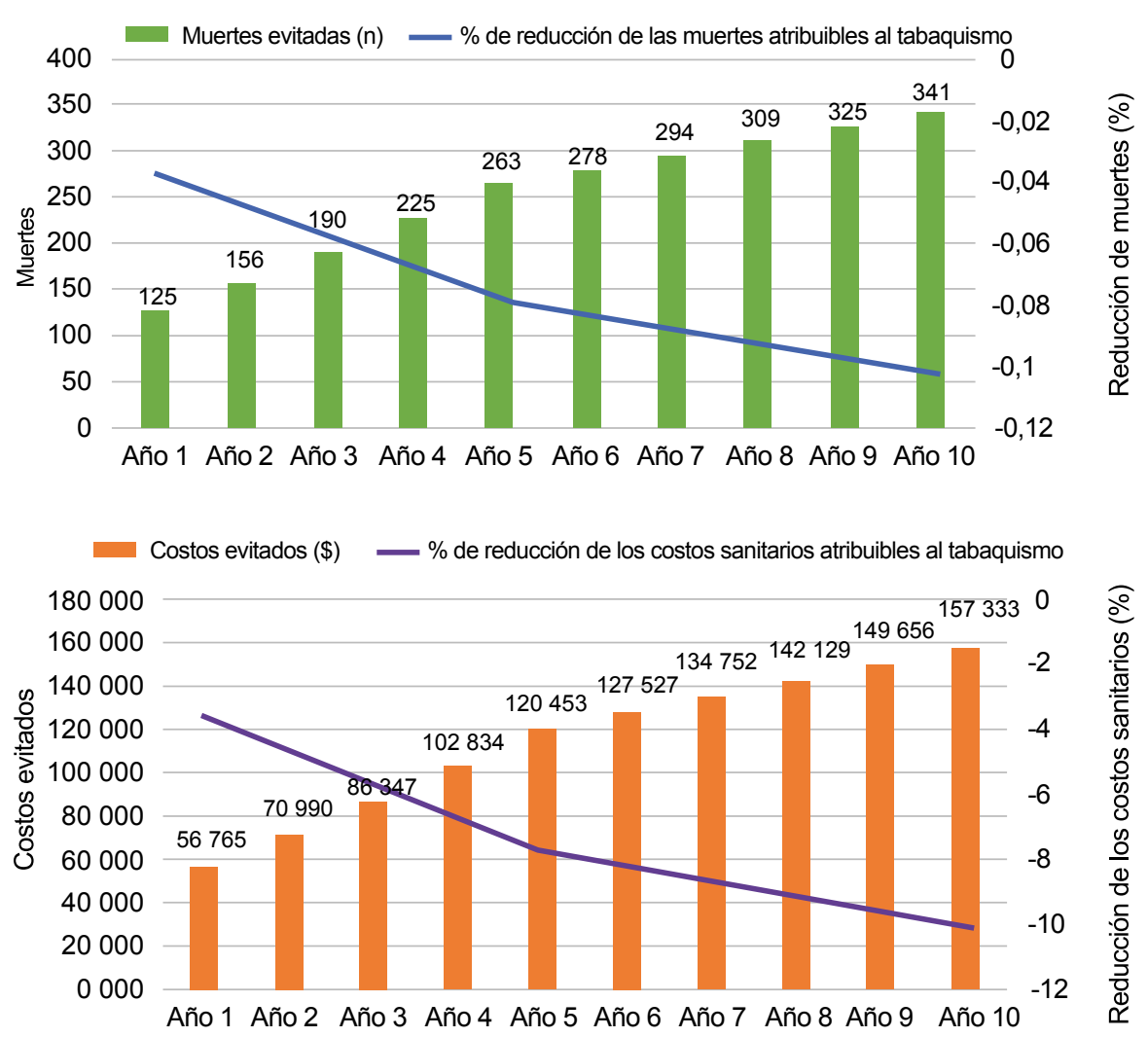

Figura 2. Escenario de aumento de precio de cigarrillos de $50 \%$ y los beneficios esperados a diez años en Paraguay, 2015

por grupos de nivel educativo o por quintiles de ingresos, pero sí se observa una mayor prevalencia de consumo a nivel urbano, en hombres y en las poblaciones con edades superiores a 30 años ${ }^{(5)}$. En este sentido, se percibe además que el consumo de tabaco entre los jóvenes está descendiendo. Esto es observado en la secuencia de las Encuestas Globales de Consumo de Tabaco en jóvenes en Paraguay realizadas en 2008 y 2014, con una prevalencia de consumo de cigarrillos de $8,3 \%$ y 5,8 \%, respectivamente ${ }^{(6,30)}$. Se estima que en Latinoamérica un aumento del $10 \%$ en los precios reduciría la demanda de cigarrillos en al menos $5 \%{ }^{(31)}$. Este u otro tipo de aumento de precios del tabaco, impactará esencialmente en la iniciación y la cesación del consumo de los jóvenes ${ }^{(32,33)}$. Esto es importante desde el punto de vista de salud pública ya que la exposición al tabaco desde la juventud y a lo largo de los años es el marcador principal del daño a la salud individual y poblacional.

Este estudio corresponde a un grupo de trabajo en Latinoamérica que busca utilizar una modalidad de decisión con fuertes evidencias científicas e información convalidadas por expertos de varios países ${ }^{(14)}$; sin embargo, presenta algunas limitaciones, puesto que es una estimación bastante conservadora debido principalmente a la falta de inclusión de una inmensa variedad de costos indirectos que podrían hasta triplicar los costos atribuibles al tabaquismo ${ }^{(10,34,35)}$. Por otra parte, cabe considerar la falta de datos precisos en Paraguay de ciertos parámetros epidemiológicos y de costos que son necesarios para el modelo, los cuales generan alguna incertidumbre en los resultados, pero que se intentan corregir con la calibración.

El cambio reciente observado en la legislación de Paraguay ha conseguido concretar un proceso de concientización sobre el impacto del consumo del tabaco que se viene trabajando desde hace años en el país. Aún es necesario profundizar en las campañas de concientización y ayuda para que los fumadores dejen de serlo. Las advertencias textuales y gráficas ayudan a reducir el consumo, así como otras medidas efectivas propuestas por la ley, como la prohibición de la publicidad, el control de la venta y el comercio, el control de los ambientes libres de humo y la trazabilidad de los productos de tabaco. Pero en lo relacionado a los impuestos, aún queda una brecha importante de ajuste que realizar.

En relación con los impuestos indirectos al tabaco, en 2015 el impuesto ad valorem fue del 6,91 \% sin impuesto indirecto específico. En relación al IVA, fue del 9,09\%, configurando un impuesto indirecto total de $16,0 \%{ }^{(9)}$. Actualmente, según el Banco Mundial, una caja de 20 cigarrillos en Paraguay 
es, después del de Irak, el más barato del mundo, con un precio de 0,54 dólares ${ }^{(8)}$. Cabe destacar que a pesar de aplicar el máximo aumento impositivo propuesto en este trabajo, el valor seguiría estando por debajo de $65 \%$ del precio de expendio que recomienda el Banco Mundial; y con precios por paquete comparables a otros países de América del Sur ${ }^{(8,32)}$.

En conclusión, las enfermedades atribuibles al tabaquismo conllevan un importante daño en Paraguay. Teniendo en cuenta que estas estimaciones son conservadoras y la realidad del daño atribuible real podría ser mucho mayor considerando costos indirectos. El equipo de investigadores espera que nuestras estimaciones lleven a aumentar la conciencia y que constituyan una base para que el gobierno y otros actores sociales logren impulsar medidas costo-efectivas.

Agradecimientos: Al Dr. Akram Abdul Hernandez-Vazquez, por su colaboración en la evaluación final del manuscrito, al bibliotecario del Instituto de Efectividad Clínica y Sanitaria Lic.
Daniel Comandé, por su ayuda con las búsquedas electrónicas y al Sanatorio y Servicios Médicos Migone (SSM Migone), de Asunción por su colaboración con la provisión de costos.

Contribuciones de autoría: APR, FA, AB, AA, JC, AP fueron responsables de la idea general y de la conducción del proyecto en varios países. JC, AA, AB, AP recopilaron los datos epidemiológicos y de costos. FCV, VGS aportaron datos primarios, ayudaron en la redacción de diferentes secciones, $A B, J C, A A, F A, A P, B R, F C V$, VGS y APR analizaron e interpretaron los datos, redactaron el manuscrito y aprobaron la versión final.

Fuentes de financiamiento: Este trabajo se realizó con la ayuda de una subvención del Centro Internacional de Investigaciones para el Desarrollo, Ottawa, Canadá (IDRC). Título del proyecto: Empowering healthcare decision makers to achieve regional needs for tobacco in Latin America: Moving forward interventions through evidence packages and cost-effectiveness evaluations (Proyecto IDRC-Componente Número-Actividad: 107978-000000000-001). Las opiniones expresadas en este documento no representan necesariamente las de IDRC o las de su Junta de Gobernanza.

Conflictos de interés: Ninguno declarado por los autores.

\section{REFERENCIAS BIBLIOGRÁFICAS}

1. Institute of Health Metrics [Internet]. Seattle: Global Burden of Disease Study; 2017. [Citado 23 Nov 2018]. Disponible en: http://www.healthdata.org/paraguay

2. Centers for Disease Control and Prevention. Smoking-attributable mortality, morbidity, and economic costs (SAMMEC). Adult SAMMEC, Relative Risk - CPS-II (82-88). Atlanta2008. Available from: http://apps. nccd.cdc.gov/sammec/.

3. Ciapponi A, Bardach A, Casetta B, Aruj P, Linetzky B, Glujovsky D, et al. Systematic review of the link between tobacco and poverty. Geneva: World Health Organization; 2011. Disponible en: http://apps.who.int/iris/bitstre $\mathrm{am} / 10665 / 44453 / 1 / 9789241500548$ eng.pdf

4. Pichon-Riviere A, Bardach A, Augustovski F, Alcaraz A, Reynales-Shigematsu LM, Pinto MT, et al. Impacto económico del tabaquismo en los sistemas de salud de América Latina: un estudio en siete países y su extrapolación a nivel regional. Rev Panam Salud Publica. 2016;40(4):213-21

5. Ministerio de Salud Pública y Bienestar Social. Primera Encuesta Nacional de Factores de Riesgo de Enfermedades No Transmisibles. Asunción, Paraguay2012. Available from: http:// www.mspbs.gov.py/dvent/wp-content/uploads/2015/10/Encuesta-Nacional.pdf.

6. Ministerio de Salud Pública y Bienestar Social. Encuesta Global de Consumo de Tabaco en Jóvenes en Paraguay 2014. . Paraguay2014. Available from: http://www.mspbs.gov.py/dvent/ wp-content/uploads/2016/01/Encuesta-Global-de-Consumo-de-Tabaco-en-J\%C3\%B3venes-2014.pdf.

7. Organización Mundial de la Salud (OMS). Convenio Marco de la OMS para el Control del Tabaco [Internet]. Ginebra: OMS; 2003. Disponible en: http://apps.who.int/iris/ bitstream/10665/42813/1/9243591010. pdf ?ua $=1$.

8. World Health Organization (WHO). WHO report on the global tobacco epidemic, 2015: raising taxes on tobacco [Internet]. Luxenburg: WHO; 2015. Disponible en: http://apps.who.int/iris/bitstr eam/10665/178574/1/9789240694606 eng.pdf ?ua $=1 \& u a=1$.

9. Organización Panamericana de la Salud (OPS). Informe sobre el Control del Tabaco en la Región de las Américas. A 10 años del Convenio Marco de la Organización Mundial de la Salud para el Control del Tabaco [Internet]. Washington, DC: OPS; 2016. Disponible en: http:// iris.paho.org/xmlui/bitstream/han- dle/123456789/28380/9789275318867_ spa.pdf ? sequen ce $=1 \&$ is Allowed $=y \& u a=1$.

10. Lightwood J, Collins D, Lapsley $\mathrm{H}$, Novotny TE. Estimating the costs of tobacco use [Internet]. En: Jha P, Chaloupka F, editors. Tobacco control in developing countries. Oxford: Oxford University Press; 2000. Disponible en: http://siteresources.worldbank.org/ INTETC/Resources/375990-108990 4539172/063TO104.PDF.

11. Guindon GE, Tobin S, Yach D. Trends and affordability of cigarette prices: ample room for tax increases and related health gains. Tob control. 2002;11(1):35-43.

12. Ross H, Chaloupka FJ. Economic policies for tobacco control in developing countries. Salud Publica Mex. 2006;48 Suppl 1:S113-20.

13. Hoffman SJ, Tan C. Overview of systematic reviews on the health-related effects of government tobacco control policies. BMC Public Health. 2015;15:744. doi: 10.1186/s12889015-2041-6.

14. Pichon-Riviere A, Augustovski F, Bardach A, Colantonio L, Latinclen Tobacco Research Group. Development and validation of a microsimulation economic model to evaluate the disease burden associated with smoking and the cost-effectiveness of tobacco 
control interventions in Latin America. Value Health. 2011;14(5 Suppl 1):S519. doi: 10.1016/j.jval.2011.05.010.

15. Alcaraz A, Caporale J, Bardach A, Augustovski F, Pichon-Riviere A. Carga de enfermedad atribuible al uso de tabaco en Argentina y potencial impacto del aumento de precio a través de impuestos. Rev Panam Salud Publica. 2016;40(4):204-212.

16. Bardach AE, Caporale JE, Alcaraz A, Augustovski F, Huayanay-Falconi L, Loza-Munarriz C, et al. Carga de enfermedad por tabaquismo e impacto potencial del incremento de precios de cigarrillos en el Perú. Rev Peru Med Exp Salud Publica. 2016;33(4):651-661. doi: 10.17843/rpmesp.2016.334.2548.

17. McLean S, Barbour V, Wild S, Simpson C, Sheikh A. Models for estimating projections for disease prevalence and burden: a systematic review focusing on chronic obstructive pulmonary disease. J J Health Serv Res Policy. 2015;20(4):246-53. doi: $10.1177 / 1355819615579232$.

18. Pinto MT, Pichon-Riviere A, Bardach A. The burden of smokingrelated diseases in Brazil: mortality, morbidity and costs. Cad Saude Publica. 2015;31(6):1283-97. doi: 10.1590/0102-311X00192013.

19. Barendregt JJ, Van Oortmarssen GJ, Vos T, Murray CJ. A generic model for the assessment of disease epidemiology: the computational basis of DisMod II. Popul Health Metr. 2003;1(1):4.

20. Ferlay J, Soerjomataram I, Dikshit R, Eser S, Mathers C, Rebelo M, et al. Cancer incidence and mortality worldwide: sources, methods and major patterns in GLOBOCAN 2012. Int J Cancer. 2015;136(5):E359-86. doi: 10.1002/ ijc.29210.

21. Lauer JA, Rohrich K, Wirth H, Charette C, Gribble S, Murray CJ. PopMod: a longitudinal population model with two interacting disease states. Cost Eff Resour Alloc. 2003;1(1):6.

22. Dirección Nacional de Estadísticas y Censos. Paraguay Proyección de la Población por Sexo y Edad, según Departamento, 2000-2025. Revisión 2015. Paraguay: DGEEC; 2015. Available from: http://www.dgeec.gov.py/Publicaciones/Biblioteca/proyeccion $\% 20$ nacional/Proyecciones\%20Departamentales\%20-\%20final.pdf.

23. Pichon-Riviere A, Alcaraz A, Bardach A, Augustovski F, Caporale J, Caccavo F. Carga de Enfermedad atribuible al Tabaquismo en Argentina. Documento Técnico $\mathrm{N}^{\circ} 7$. Buenos Aires: Instituto de Efectividad Clínica y Sanitaria (IECS); 2013. Disponible en: http://www.iecs. org.ar/wp-content/uploads/N7Carga-de-Enfermedad-Tabac-Argentina.pdf.

24. Ross H, Blecher E, Yan L, Hyland A. Do cigarette prices motivate smokers to quit? New evidence from the ITC survey. Addiction. 2011;106(3):609-19. doi: 10.1111/j.1360-0443.2010.03192.x.

25. Ministerio de Hacienda de Paraguay. Recaudación estimada de ISC de Tabaco y Cigarrillos para el año 2016. 2017.

26. San Martín V, Gamarra de Cáceres G. Mortalidad atribuible al consumo de tabaco durante los años 1998, 1999 y 2000 en Paraguay. Mem Inst Investig Cienc Salud. 2006;4(1):15-8.

27. European Commission [Internet]. Brussels: European Commission (Acceso 23 de noviembre, 2018). Excise Duties on Tobacco [dos pantallas]. Disponible en: https://ec.europa.eu/taxation_customs/ business/excise-duties-alcohol-tobacco-energy/excise-duties-tobacco

28. Chaloupka FJ, Yurekli A, Fong GT. Tobacco taxes as a tobacco control strategy. Tob Control. 2012;21(2):172-80. doi: 10.1136/tobaccocontrol-2011-050417.
29. Mathers CD, Loncar D. Projections of global mortality and burden of disease from 2002 to 2030. PLoS Med. 2006;3(11):e442.

30. Ministerio de Salud Pública y Bienestar Social. Encuesta Global de consumo de Tabaco en Jóvenes en Paraguay. Paraguay2008. Available from: http://www.mspbs.gov.py/dvent/ wp-content/uploads/2016/01/Encuesta-Global-de-Consumo-de-Tabaco-en-J\%C3\%B3venes-2008.pdf.

31. Guindon GE, Paraje GR, Chaloupka FJ. El impacto de los precios e impuestos sobre el consumo de productos de tabaco en América Latina y el Caribe. Rev Panam Salud Publica. 2016;40(4):272-284.

32. Ekpu VU, Brown AK. The Economic Impact of Smoking and of Reducing Smoking Prevalence: Review of Evidence. Tob Use Insights. 2015;8:1-35. doi: 10.4137/ TUI.S15628.

33. Kostova D. A (nearly) global look at the dynamics of youth smoking initiation and cessation: the role of cigarette prices. Applied Economics. 2013;45(28):3943-51. doi: 10.1080/00036846.2012.736947

34. Bundhamcharoen K, Aungkulanon S, Makka N, Shibuya K. Economic burden from smoking-related diseases in Thailand. Tob Control.2016;25(5):532-7.doi: 10.1136/tobaccocontrol-2015-052319.

35. Hoang Anh PT, Thu le T, Ross H, Quynh Anh N, Linh BN, Minh NT. Direct and indirect costs of smoking in Vietnam. Tob Control. 2016;25(1):96-100. doi: 10.1136/tobaccocontrol-2014-051821.

Correspondencia: Ariel Bardach

Dirección: Dr. Emilio Ravignani 2024 (C1014CPV). Buenos Aires, Argentina Correo electrónico:abardach@iecs.org.ar 


\section{ANEXOS}

Anexo 1. Costos asociados y atribuibles al tabaquismo, 2015

\begin{tabular}{lcc}
\hline Patología asociada al tabaquismo & $\begin{array}{c}\text { Costos totales } \\
\text { (millones de USD) }\end{array}$ & $\begin{array}{c}\text { Costos atribuibles } \\
\text { (millones de USD) }\end{array}$ \\
\hline $\begin{array}{l}\text { Infarto agudo de miocardio (IAM) } \\
\text { Síndrome coronario agudo no IAM }\end{array}$ & 473 & 111 \\
Muerte cardiovascular de causa no isquémica & & 19 \\
\hline Accidente cerebrovascular & 99 & 16 \\
\hline Cáncer de pulmón & 19 & 0 \\
\hline Neumonía & 0 & 76 \\
\hline Enfermedad pulmonar obstructiva crónica & 103 & \\
\hline
\end{tabular}

Cáncer de boca y faringe

Cáncer de esófago

Cáncer de estómago

Cáncer de páncreas

Cáncer de riñón

Cáncer de laringe

Leucemia

Cáncer de vejiga

Cáncer de cuello de útero

\begin{tabular}{llc} 
Tabaquismo pasivo y otras causas & NC & 31 \\
\hline Total por patologías asociadas al tabaquismo & 760 & 271 \\
\hline
\end{tabular}

* Tasa de cambio: 1 USD = 5787 guaraníes (diciembre 2015)

† El monto expresado corresponde globalmente a IAM, síndrome coronario no IAM y muerte cardiovascular de causa no isquémica

₹El monto expresado corresponde globalmente a todos los cánceres considerados

PYG: guaraníes, USD: dólares estadounidenses, NC: no corresponde. 
Anexo 2. Fuente de información de la Tabla 1

a Dirección Nacional de Estadísticas y Censos. Paraguay Proyección de la Población por Sexo y Edad, según Departamento, 20002025. Revisión 2015. Paraguay: DGEEC; 2015. Available from: http://www.dgeec.gov.py/Publicaciones/Biblioteca/proyeccion\%20 nacional/Proyecciones\%20Departamentales\%20-\%20final.pdf.

b Ministerio de Salud Pública y Bienestar Social. Primera Encuesta Nacional de Factores de Riesgo de Enfermedades No Transmisibles. Asunción, Paraguay2012. Available from: http://www.mspbs.gov.py/dvent/wp-content/uploads/2015/10/EncuestaNacional.pdf.

c Datos de mortalidad por sexo y edad. Paraguay. Ministerio de Salud Pública y Bienestar Social. 2015

d Main C, Palmer S, Griffin S, Jones L, Orton V, Sculpher M, et al. Clopidogrel used in combination with aspirin compared with aspirin alone in the treatment of non-ST-segment-elevation acute coronary syndromes: a systematic review and economic evaluation, Health Technol Assess. 2004 Oct;8(40):iii-iv, xv-xvi, 1-141.

e Nease RF Jr, Kneeland T, O'Connor GT, Sumner W, Lumpkins C, Shaw L, et al. Variation in patient utilities for outcomes of the management of chronic stable angina, Implications for clinical practice guidelines, Ischemic Heart Disease Patient Outcomes Research Team. JAMA. 1995 Apr 19;273(15):1185-90.

f Tengs TO, Yu M, Luistro E. Health-related quality of life after stroke a comprehensive review, Stroke, 2001;32(4):964-72.

$g$ Pepper PV, Owens DK. Cost-effectiveness of the pneumococcal vaccine in healthy younger adults, Med Decis Making. 2002;22(5 Suppl):S45-57.

h Hamel MB, Phillips RS, Davis RB, Teno J, Connors AF, Desbiens N, et al. Outcomes and cost-effectiveness of ventilator support and aggressive care for patients with acute respiratory failure due to pneumonia or acute respiratory distress syndrome. Am $\mathrm{J}$ Med, 2000;109(8):614-20.

i Rutten-van Molken M, Lee TA, Economic modeling in chronic obstructive pulmonary disease. Proc Am Thorac Soc. 2006;3(7):630-4.

j Montazeri A, Gillis CR, McEwen J. Quality of life in patients with lung cancer: a review of literature from 1970 to 1995 . Chest, 1998;113(2):467-81,

k Downer MC, Jullien JA, Speight PM. An interim determination of health gain from oral cancer and precancer screening: 1, Obtaining health state utilities, Community Dent Health. 1997;14(3):139-42.

I Graham AJ, Shrive FM, Ghali WA, Manns BJ, Grondin SC, Finley RJ, et al. Defining the optimal treatment of locally advanced esophageal cancer: a systematic review and decision analysis, Ann Thorac Surg. 2007;83(4):1257-64.

m Shenfine J, McNamee P, Steen N, Bond J, Griffin SM. A pragmatic randomised controlled trial of the cost-effectiveness of palliative therapies for patients with inoperable oesophageal cancer. Health Technol Assess. 2005;9(5):iii,1-121.

n Dan YY, So JB, Yeoh KG. Endoscopic screening for gastric cancer. Clin Gastroenterol Hepatol. 2006;4(6):709-16.

- Gordois A, Scuffham P, Warren E, Ward S. Cost-utility analysis of imatinib mesilate for the treatment of advanced stage chronic myeloid leukaemia. Br J Cancer, 2003;89(4):634-40.

p Heinzer H, Mir TS, Huland E, Huland $\mathrm{H}$. Subjective and objective prospective, long-term analysis of quality of life during inhaled interleukin-2 immunotherapy. J Clin Oncol. 1999 Nov;17(11):3612-20.

q Stratton KR, Durch JS, Lawrence RS, editors. Vaccines for the 21st Century. Washington (DC): National Academies Press; 2000.

$r$ Sung L, Buckstein R, Doyle JJ, Crump M, Detsky AS. Treatment options for patients with acute myeloid leukemia with a matched sibling donor. Cancer, 2003;97(3):592-600.

S Ingresos nacionales por impuestos al tabaco: Ministerio de Hacienda de Paraguay, Recaudación estimada de ISC de Tabaco y Cigarrillos para el año 2016.

t Producto bruto interno: World Bank, World Development Indicators; 2015.

u Guindon GE, Paraje GR, Chaloupka FJ. El impacto de los precios e impuestos sobre el consumo de productos de tabaco en América Latina y el Caribe. Rev Panam Salud Publica. 2016;40(4):272-284.

v World Bank, World Development Indicators, 2015, 